\title{
La verdad en la literatura médica
}

\section{Truth in medical literature}

\author{
Mónica Zuluaga-Quintero • Medellín (Colombia)
}

DOI: https://doi.org/10.36104/amc.2021.1859

\section{Resumen}

Diferentes características influyen en la publicación de artículos científicos, dentro de los cuales se destaca: la calidad del contenido, la redacción, el tema de preferencia de la revista y las necesidades del momento de acuerdo con los avances o problemáticas en salud. Sin embargo, otros aspectos como el tipo de resultados de la investigación, el reconocimiento de los autores y las preferencias editoriales marcan la decisión de rechazar o aceptar un manuscrito; conocidos como sesgos de publicación. Reconocerlos permite a docentes, investigadores y estudiantes mantener la motivación y el juicio clínico para plantear propuestas académicas relevantes y tener un análisis crítico del conocimiento. (Acta Med Colomb 2021; 46. DOI: https://doi.org/10.36104/amc.2021.1859).

Palabras clave: sesgo de publicación, investigación, ética en la publicación científica, educación médica.

\begin{abstract}
Various characteristics influence the publication of scientific articles, including the quality of the content, its drafting, the journal's preferred topic, and the current needs, according to health advances or issues. However, other aspects such as the type of research results, the authors' recognition and editorial preferences mark the decision to reject or accept a manuscript; these are known as publication bias. Recognizing these biases allows teachers, researchers and students to maintain motivation and clinical judgement for presenting relevant academic proposals, and maintain a critical analysis of knowledge. (Acta Med Colomb 2021; 46. DOI: https://doi.org/10.36104/amc.2021.1859).

Key words: publication bias, research, ethics in scientific publication, medical education.
\end{abstract}

Dra. Mónica Zuluaga-Quintero: Internista, Master en Metodología de la Investigación en Ciencias de la Salud, Epidemióloga Universidad Autónoma de Bucaramanga. Internista Salud Sura Industriales, Hospital Pablo Tobón Uribe. Medellín (Colombia).

Correspondencia: Dra. Mónica ZuluagaQuintero. Medellín (Colombia).

E-Mail: mzulu28@hotmail.com Recibido: 03/V/2020 Aceptado: 9/XII/2020
En los últimos años el número de publicaciones científicas ha incrementado en paralelo con la celeridad para el acceso a la información a través de la red, la existencia de nuevas bases de datos y revistas electrónicas, que surgen en respuesta a la demanda que la globalización conlleva. Esto tiene consecuencias tanto positivas como negativas para el mundo científico y en particular el sector salud; pues si bien hay mayor acceso a la información y a su vez más evidencia, no siempre esto refleja datos verídicos y precisos, los cuales se deben juzgar de manera correcta por el público (1). En primera medida se debe tener consciencia de que: "todo lo que se publica no siempre es cierto" y a su vez que: "lo que no se publica no siempre es falso o carente de valor", pues detrás de la presentación de un manuscrito en una revista científica, existen varios puntos a tener en cuenta para la decisión de ser aceptado y publicado en un momento del tiempo $(1,2)$. En general tres aspectos clave:
1. El tipo de manuscrito, tema en cuestión y calidad del mismo.

2. Los autores del manuscrito y su filiación.

3. La revista en la cual se publica y los editores de la misma.

Si bien en el escenario ideal, todo texto sometido a publicación debería ser calificado y aceptado por su contenido, es decir, el fondo. Existen aspectos subyacentes que están en medio de la decisión y que dan igual importancia a la forma; es decir, lo que está de lado al contenido como: el idioma utilizado, el reconocimiento de los autores que publican, las filiaciones de los autores a ciertas instituciones o gremios, el patrocinio de casas comerciales, la procedencia de los autores y en algunos casos la capacidad de pago para ciertas revistas que solicitan remuneración por publicaciones (3). Por su parte también existe el concepto del grupo editorial que en algunas situaciones puede verse no sólo orientado por el contenido académico 
sino también por el reconocimiento de los autores que envían los manuscritos o las necesidades propias de la revista. Todo lo anterior es a lo que llamamos sesgos en la publicación de ediciones científicas, los cuales pueden ser sesgos de publicación o editoriales (2). Afirmar que esto último ocurre en todos los casos, sería atrevido, pues desconozco el funcionamiento de cada revista en particular y sus criterios para la aceptación de artículos; e indiscutiblemente hay situaciones particulares que influyen en la toma de decisiones y que es difícil juzgar entre lo correcto o no, pues está a consideración la visión académica, la ética y la sociocultural.

Poder afirmar que una revista es mejor por recibir sólo los artículos con mayor validez científica no sería adecuado, pues determinar la calidad de un artículo o su relevancia sólo por este aspecto no es posible. Hay estudios o narrativas que no tienen el mejor nivel de evidencia, pero resultan útiles por ser la única información existente (2, 4). Un ejemplo para este caso, lo planteo con la situación vigente respecto al COVID-19. En el momento de pandemia hay más número de publicaciones respecto al tema y posiblemente la mayoría de ellas son reportes, trabajos descriptivos o ensayos no aleatorizados que no tienen el mejor nivel de evidencia, pero que son completamente útiles para la situación de salud que vivimos, donde se requiere información. Esto debió influir en la decisión de aceptar manuscritos de este tema por encima de otros artículos que hubiesen estado en espera en muchas revistas, pero las necesidades del momento ameritan su prioridad y es un sesgo de publicación que tiene un sustento teórico, social, práctico e incluso ético, si lo vemos como beneficencia y equidad al ser un tema que compete al mundo entero. De manera similar puede ocurrir con publicaciones que se presentan en revistas locales, en las cuales puede aceptarse artículos de interés nacional y en el idioma propio del país $(2,5)$; sin embargo, esto no es errado siempre y cuando se haga en beneficio del sistema de salud, puesto que es relevante la descripción de datos propios, siendo este uno de los problemas que encontramos muchas veces con ciertos estudios internacionales que no tiene validez externa y no son aplicables a nuestro medio (5).

Por su parte situaciones como la decisión de publicación según resultados positivos, el incentivo económico y el prestigio de ciertos autores; sí desestimula la iniciativa de muchos profesionales que tienen intención de impactar en salud a través de su opinión y experiencia, lastimosamente frenados por este fenómeno de comercialización que aplican algunos medios $(6,7)$. Caso particular que menciono por la experiencia desde la formación profesional, cuando se ha rechazado y luego aceptado un texto al modificar los autores y adicionar un nombre reconocido, sin cambiar el fondo del artículo. Del mismo modo ocurre con la filiación a instituciones de mayor reconocimiento o sólo por la traducción a otro idioma (2). Esto ha generado el pensamiento erróneo en muchos estudiantes que consideran que sólo puede trabajarse en investigación con ciertos docentes particulares o grupos de investigación específicos, muchas veces minorizando sus ideas y en consecuencia trabajando en proyectos que no los motivan y disminuye la capacidad de cuestionarse o generar nuevos planteamientos. Es un reto para nosotros como médicos especialistas y docentes en salud, cambiar este paradigma, motivar a los estudiantes a plantear problemas desde su propia observación e interés; a cuestionarse en el día a día de su práctica profesional y optimizar la capacidad de investigación, análisis y publicación, dando importancia al conocimiento global y no sólo al limitado por un grupo especial. El alumno que se sienta motivado a investigar, debe empoderarse de su trabajo, ser propositivo, ejecutivo y darle valor a su autoría, sólo así podremos crear una cultura de investigación objetiva y ética en el país.

Diferentes instituciones han reaccionado en respuesta a esta situación creando registros de base de datos que permitan acceder a la mayoría de publicaciones. La Organización Mundial de la Salud (OMS) ha creado la Plataforma Internacional de Registro de Ensayos Clínicos (PIREC) y propone la utilización del Número Universal de Referencia para Ensayos Clínicos o UTRN (derivado del inglés Universal Trial Reference Number). Igualmente clinicaltrials.gov, pertenece al Instituto de Salud de los Estados Unidos, es de acceso libre e incluye todos los aspectos metodológicos de los ensayos y si al momento actual el estudio terminó, se suspendió o está abierto el reclutamiento. También en Europa existe el www. controlled-trials.com, un meta-registro de ensayos clínicos controlados, destacando entre otras el International Standard Randomised Controlled Trial Number Register (ISRCTN Register) similar a la Colaboración Cochrane con el Cochrane Central Register of Controlled Trials (CENTRAL) (6).

Es adecuado y necesario que cada revista y grupo editorial tenga sus preferencias por ciertos tipos de artículos, puesto que en ello se basa el ordenamiento sistemático, pues sería imposible abarcar todo un conjunto de temas y población en un solo sector. Se requiere estadificar o estratificar las publicaciones por categorías de interés para poder organizar el conocimiento. No obstante, es importante que se tengan en cuenta los sesgos que existen y con base en ellos, el lector de manera crítica seleccione la información que considere relevante para su práctica diaria y haga un uso adecuado del recurso. En este aspecto es fundamental el rol que podemos tener los médicos tanto para hacer uso de la evidencia en nuestro ejercicio profesional, así como alertar a los alumnos a ser lectores críticos. Por su parte los comités editoriales y autores deben tener en cuenta en su selección el tema que sea representado por la revista particular, las necesidades del medio donde se publica y lo más importante la calidad de la información que se va a compartir, pues así es más factible adecuar la investigación y las publicaciones a la 
situación social y cultural propia, convirtiéndola en una verdadera ayuda para el sector salud y no en la excusa para solventar los intereses de unos pocos.

\section{Referencias}

1. Manterola C, Otzen T. Los sesgos en investigación clínica. Int.J.Morphol. 2011; 33(3):1156-1164.

2. Matias J, García R. Sesgos en la edición de publicaciones científicas. Neurología. 2011;26 (1):1-5
3. Restrepo M, Restrepo C. Sesgos en diseños analíticos. Revista Colombiana de Psiquiatría. 2004;33(3):327-335.

4. Bosques F, Gómez D. El sesgo de publicación es un problema científico frecuente que tiene consecuencias éticas adversas Medicina Universitaria 2009;11(42):1-2.

5. Campillo C. Sesgos de publicación, valor de la información y su efecto en las políticas de salud. Revista Cubana de Salud Pública 2012; 38(5): 714-724.

6. Calvo A.El registro de ensayos clínicos: o ¿Cómo evitar el sesgo de publicación? Rev Med Hered. 2007; 18(2): 57-58.

7. Baladia E, Martinez R. Sesgo de publicación: ¿QQué pueden hacer las revistas científicas? Revista Española de Nutrición Humana y Dietética. 2015; 19(3): $130-131$. 